\title{
Assembly-Controlled Permeability of Layer-by- Layer Polymeric Microcapsules Using a Tapered Fluidized Bed
}

Ka Fung Noi, ${ }^{\dagger}$ Ali Roozmand, ${ }^{\dagger}$ Mattias Björnmalm, ${ }^{\dagger}$ Joseph J. Richardson, ${ }^{\dagger, \ddagger}$ George V. Franks, ${ }^{\S}$ and Frank Caruso ${ }^{\dagger, *}$

${ }^{\dagger}$ Australian Research Council (ARC) Centre of Excellence in Convergent Bio-Nano Science and Technology, and the Department of Chemical and Biomolecular Engineering, The University of Melbourne, Parkville, Victoria 3010, Australia.

${ }^{\S}$ Department of Chemical and Biomolecular Engineering, The University of Melbourne, Parkville, Victoria 3010, Australia.

Keywords: layer-by-layer, nanofilm properties, swelling, permeability, microcapsule 


\section{ABSTRACT}

Nano- and microcapsules engineered through layer-by-layer (LbL) assembly are finding an increasingly large number of applications as catalysts, electrochemical biosensors, bioreactors, artificial cells and drug delivery vehicles. While centrifugation-based LbL assembly is the most common method for coating template particles and preparing capsules, it is a batch process and requires frequent intervention that renders the system challenging to automate and scale up. Here, we report the use of a tapered fluidized bed (TFB) for the preparation of multilayered polymer capsules. This is a significant improvement over our recent approach of fluidizing particles in cylindrical fluidized beds (CFB) for LbL assembly. We demonstrate that TFB is compatible with particles $<3 \mu \mathrm{m}$ in diameter (an order-of-magnitude improvement compared with CFB), which can be fluidized with minimal entrainment. Additionally, layering materials were expanded to include both electrostatic and hydrogen-bonding polymer pairs (e.g., poly(allylamine hydrochloride) (PAH) and poly(styrene sulfonate) (PSS), and thiol-modified poly(methacrylic acid) (PMAsH) and poly(N-vinylpyrrolidone) (PVPON), respectively). Finally, differences between capsules prepared via centrifugation-based and TFB LbL assembly were investigated. The obtained TFB microcapsules demonstrate increased film thickness and roughness compared with those prepared using centrifugation-based LbL assembly. Furthermore, $\mathrm{PMA}_{\mathrm{SH}}$ microcapsules exhibit lower swelling and permeability when prepared via TFB LbL assembly compared with centrifugation-based LbL assembly due to enhanced multilayer deposition, entanglement and cross-linking. Therefore, polymeric capsules fabricated via TFB LbL assembly may be useful for encapsulation and retention of relatively low molecular weight ( 20 kDa) hydrophilic biomacromolecules to passively or responsively release the payload for potential drug delivery application. 


\section{INTRODUCTION}

Layer-by-layer (LbL) assembly has drawn growing scientific interest over the past two decades as a versatile approach to assemble functional polymeric nanofilms on a wide range of templates. ${ }^{1,2}$ While centrifugation-based LbL assembly has a broad toolkit with regard to choice of templates (e.g., gold, ${ }^{3}$ silica, ${ }^{4}$ emulsions ${ }^{5}$ ) and materials (e.g., polymers, ${ }^{6}$ biomolecules, ${ }^{7}$ DNA, $^{8} \quad$ colloids $^{9}$ ), it generally requires numerous cycles of (nonflow) adsorption/centrifugation/wash steps to remove excess materials. This inherently limits centrifugation-based LbL assembly, as it is labor-intensive, time consuming, and the use of small templates can lead to aggregation issues under high-speed centrifugation. To address these limitations, a variety of LbL assembly techniques have been developed, including, immersive assembly, ${ }^{1}$ membrane filtration, ${ }^{10}$ spray assembly, ${ }^{11}$ spin assembly, ${ }^{12}$ atomization, ${ }^{13}$ immobilization, ${ }^{14}$ microfluidic assembly, ${ }^{15}$ and electrophoretic assembly. ${ }^{16}$ However, these techniques often reduce the diversity of applicable materials and particles that can be employed, or tend to introduce complex and specialized equipment, or reduce the potential for scaling up production. $^{2}$

Recently, we reported the concept of fluidizing particle templates in a cylindrical fluidized bed (CFB) for rapid and versatile layering. ${ }^{17}$ While the fabrication of $30-50 \mu \mathrm{m}$ microcapsules in relatively large quantities was successful, fluidizing smaller-sized particles $(\sim 5 \mu \mathrm{m})$ was difficult due to entrainment at relatively high flow rates. Herein, we report the use of a tapered fluidized bed (TFB) for layering fluidized smaller particle templates and fabricating polymer capsules with controlled properties. TFBs have been widely applied in many industrial processes such as particle segregation, ${ }^{18}$ combustion, ${ }^{19}$ wastewater treatment, ${ }^{20}$ and bioreactors. ${ }^{21}$ Similar to a CFB, an upward flow of fluid is used to fluidize particle templates. Whereas CFBs have a constant 
cross-sectional column, TFBs are in the form of a cone with small, cross-sectional entrances and a gradual expansion of cross-sectional area along the bed heights from the bottom to the top (Figure 1). Therefore, there is a velocity gradient where the velocity of the fluid is relatively high at the bottom with decreasing velocity at the top, preventing the entrainment of small particles. ${ }^{22}$ This velocity gradient circumvents the entrainment issue experienced when layering smaller particles using $\mathrm{CFB}$, while also maintaining continuous layering and eliminating the need for centrifugation and washing steps common to centrifugation-based LbL assembly (Figure 2). Additionally, the material choice for fluidized bed layering was expanded by utilizing both electrostatic and hydrogen bonding complementary polymers, and the film properties were shown to be different from those prepared by centrifugation-based LbL assembly. Hence, TFB LbL assembly possesses the capability to: i) operate over a wide range of fluid velocities, ii) fluidize microparticles $(\sim 3 \mu \mathrm{m})$ at high flow rates with minimal entrainment, iii) facilitate the layering of polymers through electrostatic forces and hydrogen bonding, and iv) manipulate material properties such as film roughness, degree of swelling, and permeability. TFB LbL assembly therefore addresses key challenges associated with CFB LbL assembly, while retaining advantages such as ease-of-use, high amenability to automation, and potential for scale-up. 

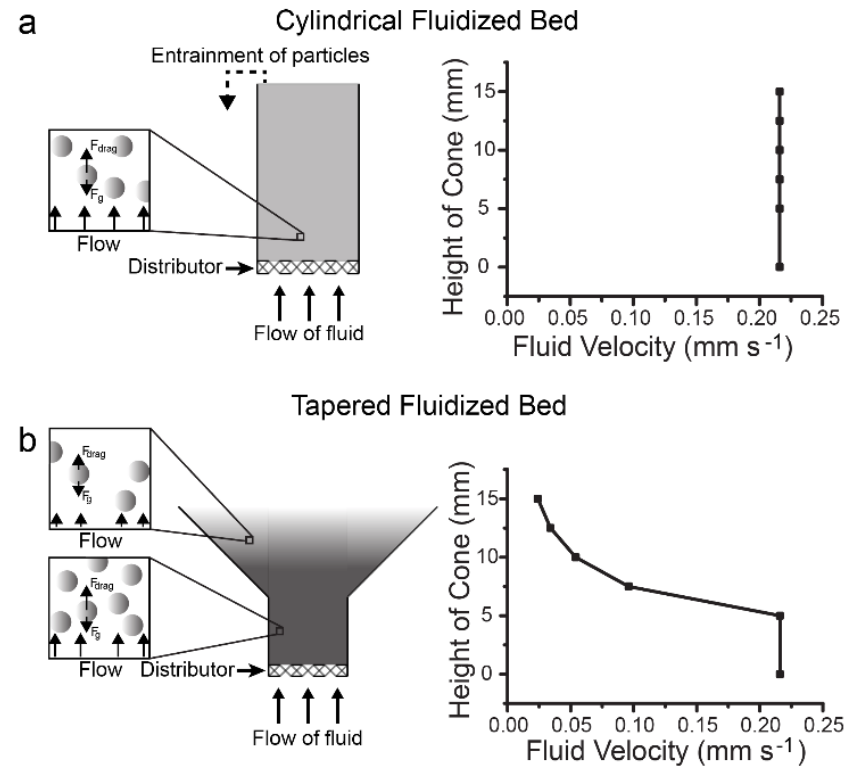

Figure 1. Schematic view (not to scale) of (a) cylindrical and (b) tapered fluidized beds for the continuous LbL deposition of polymers for the generation of core-shell particles and polymer capsules.

a) Centrifugation-based LbL Assembly

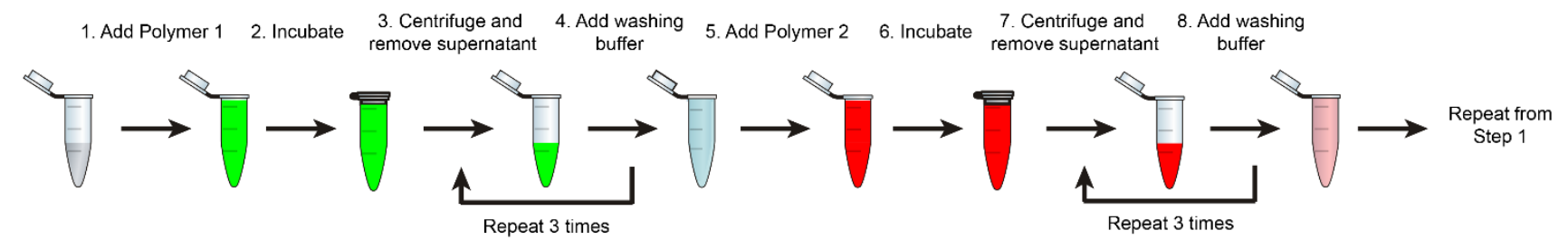

b) Tapered Fluidized Bed LbL Assembly

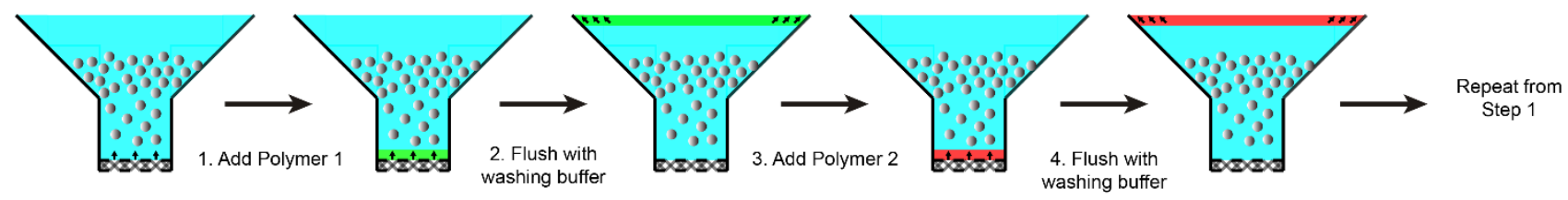

Figure 2. Core-shell particles prepared via centrifugation-based (a) and tapered fluidized bed (b) LbL assembly. Centrifugation-based LbL assembly is a batch-wise process involving multiple centrifugation-wash steps and long incubation times, whereas TFB LbL assembly is an upward 
flow process with fewer steps to achieve bilayer deposition, which eliminates the need for centrifugation-wash steps.

\section{EXPERIMENTAL SECTION}

Fluidization of Template Particles. Ultrapure water with a resistivity greater than $18 \mathrm{M} \Omega . \mathrm{cm}$, from an inline Millipore RiOs/Origin purification system, was used. $500 \mu \mathrm{L}$ of particles $(7 \mu \mathrm{m}$ silica, 5 wt \%) from Microparticles $\mathrm{GmbH}$, was added into the fluid bed column $(1 \mathrm{~cm}$ in diameter NAP-5 column from GE Healthcare Life Sciences), and the pump (PHD2000 from Harvard Apparatus) flow rate was set to $1000 \mu \mathrm{L} \mathrm{min}{ }^{-1}$.

Experiments were performed with a tapered column, $10 \mathrm{~mm}$ in length, and an overall taper angle of $45^{\circ}$. The column size increased from $10 \mathrm{~mm}$ at the bottom to $30 \mathrm{~mm}$ at the top (Figure S1).

Preparation of Thiol-Functionalized Poly(methacrylic acid) (PMA ${ }_{S H}$ ). Poly(methacrylic acid) with 12 mol \% thiol modification was synthesized from PMA and 2-(pyridylthio)ethylamine hydrochloride via carbodiimide coupling, as described previously. ${ }^{23}$

Fluidized Bed Layer-by-Layer Capsule Assembly. The fluidization of $25 \mathrm{mg}$ of template particles was achieved using a flow rate of $1000 \mu \mathrm{L} \mathrm{min}^{-1}$ with ultrapure water. A six-port valve (IDEX Health and Science) was used for injecting the polymers (poly(sodium 4styrenesulfonate) (PSS, $\mathrm{M}_{\mathrm{W}} 70 \mathrm{kDa}$ ) and poly(allylamine hydrochloride) (PAH, $\mathrm{M}_{\mathrm{W}} 58 \mathrm{kDa}$ ), or $\mathrm{PMA}_{\mathrm{SH}}\left(\mathrm{M}_{\mathrm{W}} 15 \mathrm{k} \mathrm{Da}\right)$ and poly(N-vinylpyrrolidone) (PVPON, $\left.\mathrm{M}_{\mathrm{W}} 10 \mathrm{kDa}\right)$ ) into the fluidized bed without disrupting the flow rate of water. A polyether ether ketone tube with dimensions of $115 \mathrm{~cm}$ in length and $1.02 \mathrm{~mm}$ in inner diameter ( 1 $\mathrm{mL}$ volume) was used for loading the polymer solutions. PAH and PSS were prepared in $0.5 \mathrm{M} \mathrm{NaCl}, \mathrm{pH} 7$, with a polymer concentration of $1.0 \mathrm{mg} \mathrm{mL}^{-1}$. 
The polymer solution was injected into the loading tube using a syringe with the six-way valve set to "load", isolating the polymer solution from the flow of water. $2 \mathrm{~mL}$ of polymer solution was loaded through the $1 \mathrm{~mL}$ tube to minimize dilution of polymer solution. The valve was then set to "inject", diverting the flowing water through the polymer loading tube. The loaded polymer solution was then part of the flow stream and flowed through the fluidized bed. An additional $3 \mathrm{~mL}$ of ultrapure water was allowed to flow through the loading tube and fluidized bed to ensure that both were sufficiently washed before the valve was reset to "load" for the second polymer and the process was repeated until eight layers were deposited.

Modified Reaction Parameters. The experimental conditions were the same as the standard conditions mentioned above except for the differences specifically mentioned below. Low polymer injection flow rate: The flow rate was decreased from $1000 \mu \mathrm{L} \mathrm{min}{ }^{-1}$ to $100 \mu \mathrm{L} \mathrm{min}^{-1}$, increasing the polymer-particle contact time from 1 to $10 \mathrm{~min}$. High polymer concentration: The polymer solution concentration was increased from 1 to $4 \mathrm{mg} \mathrm{mL}^{-1}$. High polymer injection volume: The volume of polymer solution injected was increased from 1 to $2 \mathrm{~mL}$, doubling the residence time of the polymer. High washing volume: Washing volume injected was increased from 3 to $5 \mathrm{~mL}$ to ensure overflow of polymer solution and removal of free polymers.

Cross-linking of PMA PM $_{\mathrm{sH}}$ Core-Shell Particles. To stabilize the (PVPON/PMA $\mathrm{SH}_{4} / \mathrm{PVPON}$ multilayers, the coated particles were exposed to a $2.75 \mathrm{mM}$ solution of chloramine $\mathrm{T}$ (CaT) for 10 min, as described previously. ${ }^{23}$

Core Removal. The layered particles were collected into $1.7 \mathrm{~mL}$ microcentrifuge tubes, centrifuged, and the supernatant was removed. $500 \mu \mathrm{L}$ of hydrofluoric acid (5 M HF) was added to the layered particles, and incubated for 5 min to remove the silica. [Caution! HF is highly toxic. Extreme care should be taken when handling HF solution.] The capsules were pelleted at 
$2000 \mathrm{~g}$ for $3 \mathrm{~min}$, the supernatant was removed, and the capsules were washed with ultrapure water three times to ensure that the HF was fully removed.

Additional experimental details are available in the Supporting Information.

\section{RESULTS AND DISCUSSION}

Fluidization Performance of CFB and TFB. Firstly, we investigated the performance difference between CFB and TFB systems. The minimum fluidizing velocity $\left(\mathrm{U}_{\mathrm{MF}}\right)$ is the minimum velocity at which a settled bed of particles can be fluidized and the terminal velocity $\left(\mathrm{U}_{\mathrm{T}}\right)$ is the maximum velocity of a particle before it is lost from the fluidized bed due to entrainment. These velocities were calculated according to Richardson et al. ${ }^{17}$ These values were converted to minimum $\left(\dot{\mathrm{V}}_{\mathrm{MF}}\right)$ and maximum volumetric flowrates $\left(\dot{\mathrm{V}}_{\mathrm{T}}\right)$ for the fluid bed

and used as the basis of comparison and provided a guideline on expected flow rates, which are compatible with different particle sizes (Figure 3). To determine whether the $\dot{\mathrm{V}}_{\mathrm{T}}$ values were experimentally valid, $25 \mathrm{mg}(500 \mu \mathrm{L})$ of $7 \mu \mathrm{m} \mathrm{SiO}_{2}$ was fluidized at 200,400 and $600 \mu \mathrm{L} \mathrm{min}{ }^{-1}$ for CFB and TFB systems. After fluidizing the particles for a minimum of $15 \mathrm{~min}$, it was observed that the CFB system had a significant amount of particle loss whereas particles in the TFB had minimal entrainment (Figure 4). This matches closely with the calculated $\dot{V}_{\mathrm{T}}$ values of $174 \mu \mathrm{L} \min ^{-1}$ and $1270 \mu \mathrm{L} \min ^{-1}$ for $\mathrm{CFB}$ and $\mathrm{TFB}$, respectively, suggesting an accurate maximum flow rate for fluidization. The results demonstrate that TFB, with its increasing crosssectional area provides greater operation volumetric flow rate while maintaining minimum particle entrainment. Figure 5 shows the three-dimensional (3D) spatial distribution of $7 \mu \mathrm{m} \mathrm{SiO} \mathrm{Si}_{2}$ under different flow rates. It can be seen that most particles are concentrated near the vertical section of the fluidized bed with few particles near the top of the tapered column at $1000 \mu \mathrm{L} \mathrm{min}^{-1}$. 


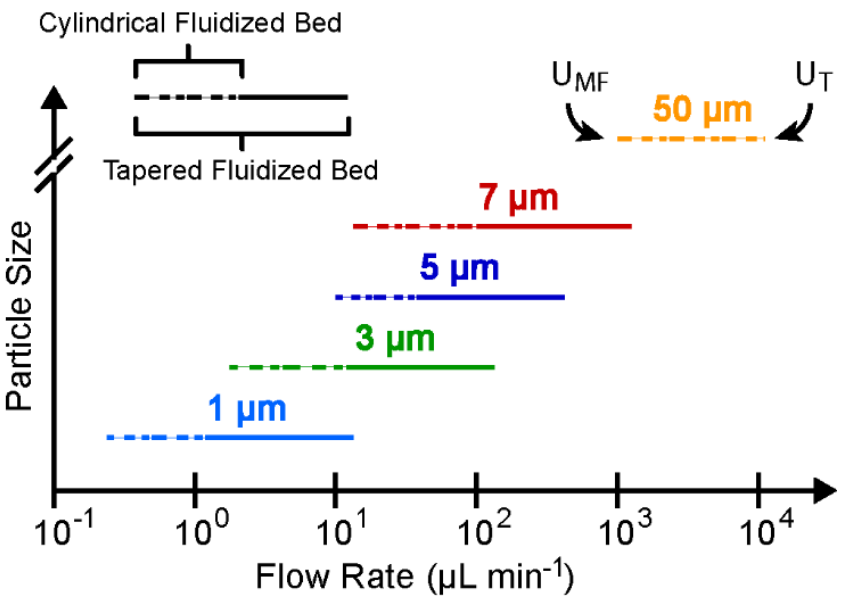

Figure 3. Comparison between cylindrical (dashed line) and tapered fluidized bed (dashed and solid line) of theoretical fluidization ranges for silica particles of different diameters. See Table S1 in Supporting Information for values.

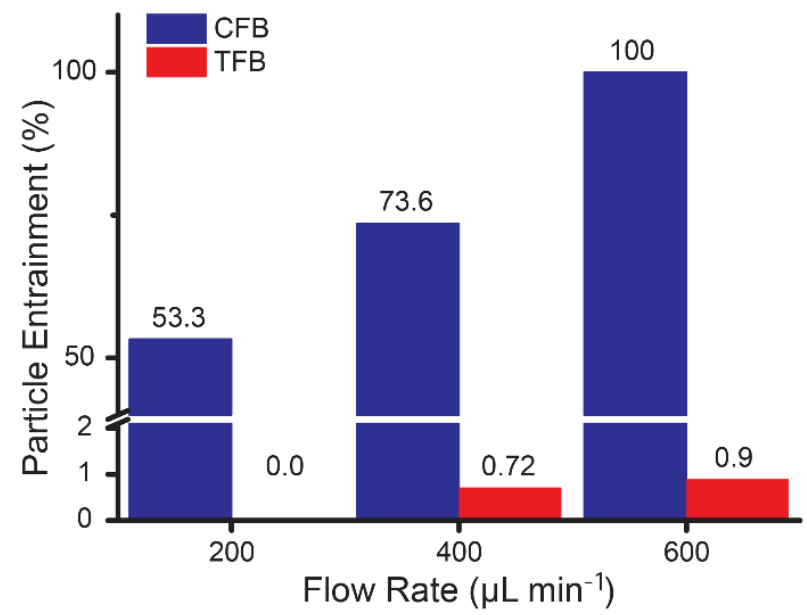

Figure 4. Percentage of particle (7 $\left.\mu \mathrm{m} \mathrm{SiO}_{2}\right)$ entrainment using $\mathrm{CFB}$ (blue) and TFB (red) at 200, 400 and $600 \mu \mathrm{L} \mathrm{m^{-1 }}$ after a minimum of 15 min of fluidization.

To further verify that the calculated $\dot{\mathrm{V}}_{\mathrm{T}}$ values for TFB are the upper limits of fluid velocities that will retain particles within the system, 2.78, 4.62 and $7 \mu \mathrm{m} \mathrm{SiO}_{2}$ were fluidized from $200 \mu \mathrm{L}$ $\min ^{-1}$ and were increased to their respective $\dot{\mathrm{V}}_{\mathrm{T}}$ value in increments of $200 \mu \mathrm{L} \mathrm{min}{ }^{-1}$ (Table S2). The number of initial particles that were entrained was recorded by bright-field microscopy and 
subsequently analyzed. Table S2 demonstrates that $\dot{\mathrm{V}}_{\mathrm{T}}$ values closely match the experimental results, where particle entrainment is evident above the terminal velocity of fluid flow rate for all sizes of $\mathrm{SiO}_{2}$ particles.

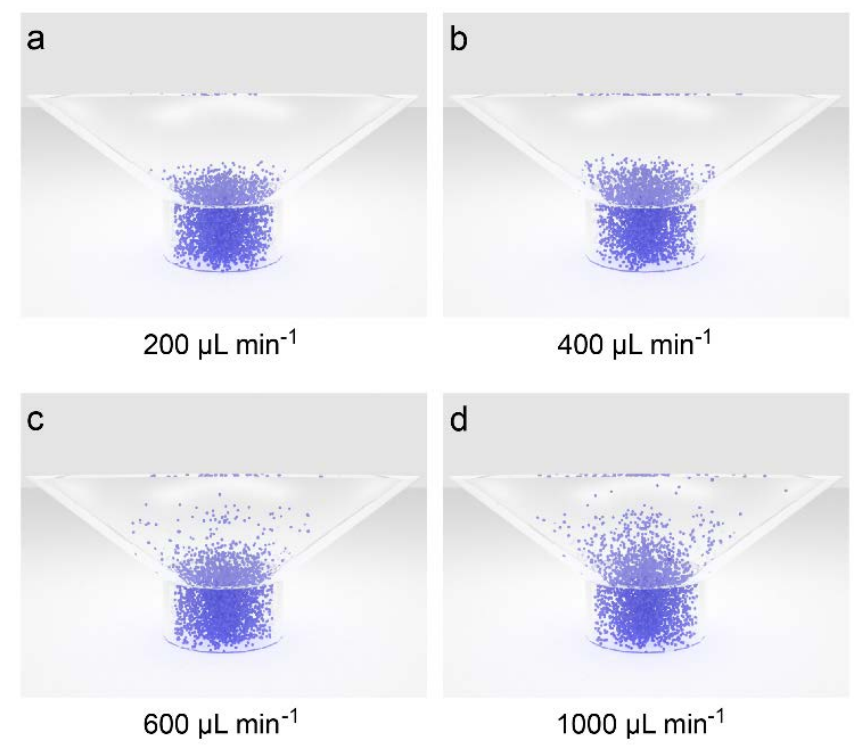

Figure 5. 3D visualization based on data of particle distributions in a TFB system at (a) 200, (b) 400, (c) 600, and (d) $1000 \mu \mathrm{L} \min ^{-1}$ for $7 \mu \mathrm{m} \mathrm{SiO}_{2}$ particles. See Supporting Methods in Supporting Information for procedure used to generate 3D visualizations.

TFB LbL-Assembled PAH/PSS Multilayers. The initial layering conditions of (PAH/PSS) $)_{4}$ were performed in accordance with Richardson et al. ${ }^{17}$ high $\mathrm{M}_{\mathrm{w}} \mathrm{PAH}$ (58 kDa) and PSS (70 kDa), polymer concentrations of $1 \mathrm{mg} \mathrm{mL}^{-1}$ and a flow rate of $1000 \mu \mathrm{L} \mathrm{min}{ }^{-1}$. DIC microscopy images show dispersed core-shell particles and no visible polymer complexes. However, after HF treatment a low number of capsules remained (Figure S2a). Zeta ( $\xi$ )-potential analysis of the layered particles, conducted in water, showed that the mean $\xi$-potential did not alternate between negative and positive with each additional layer, indicating incomplete layer and/or non-uniform deposition (Figure 6a). To improve layering, four parameters: i) polymer injection flow rate, ii) polymer concentration, iii) injected polymer volume, and iv) injected 
washing volume were optimized. Layering was improved by using a higher polymer concentration (from 1 to $4 \mathrm{mg} \mathrm{mL}^{-1}$ ), resulting in the formation of some capsules, with polymer complexes, fragmented capsules and aggregation also present (Figure S2b). By decreasing the polymer injection flow rate (from 1000 to $100 \mu \mathrm{L} \mathrm{min}{ }^{-1}$ ), which increases polymer-particle contact time (from 1 to $10 \mathrm{~min}$ ), some deformed capsules and a large number of polymer complexes were formed (Figure S2c). As both lower polymer injection flow rate and higher polymer concentration showed improvements, we examined the synergistic effect of the combination of the two, which resulted in a vast improvement in the yield and quality of the capsules formed (Figure S2d). However, free polymer complexes could still be observed, which was attributed to an insufficient volume of washing fluid to remove free polymer from the TFB. Finally, to determine if layering could be further improved, high polymer injection volume (from 1 to $2 \mathrm{~mL}$ ) and washing buffer volume (from 3 to $5 \mathrm{~mL}$ ) were employed in conjunction with high polymer concentration and low polymer injection flow rate (Figure S2e), which produced capsules closely resembling those assembled through centrifugation-based LbL assembly (Figure S2f). Multilayered particles assembled with the modified TFB LbL assembly displayed characteristic alternating $\xi$-potentials after each successive layering of PAH and PSS (Figure 6b). The fluidized bed assembly provides a way of more rapidly depositing layers of polymer on particle templates, and is approximately three times quicker than centrifugation-based LbL assembly (10 min versus $\sim 30$ min per layer and wash). 

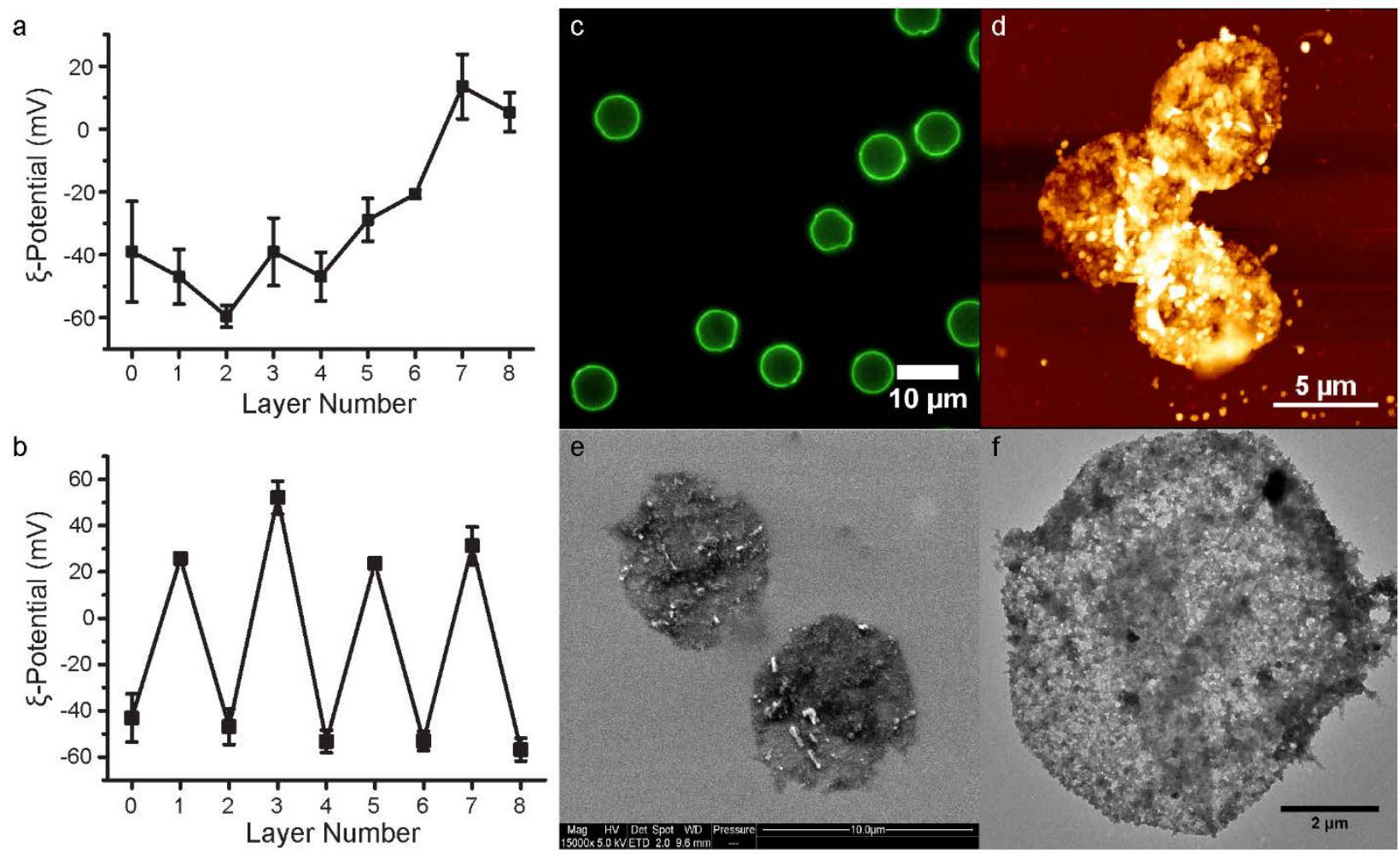

Figure 6. $\xi$-potential of reference conditions (a) and modified conditions (b) of TFB LbL assembly of PAH/PSS, where zero, odd layer and even layer number correspond to bare $7 \mu \mathrm{m}$ $\mathrm{SiO}_{2}$ particles, PAH and PSS, respectively. Fluorescence microscopy images (c), AFM images (d), SEM images (e), and TEM images (f) of FITC-dextran-labeled (green) PAH/PSS capsules prepared by TFB LbL assembly.

Hydrogen-Bonding Facilitated TFB LbL-Assembled Multilayers. After optimizing the layering conditions, we explored the versatility of TFB LbL assembly for depositing polymers through hydrogen bonding interactions. Commonly used hydrogen donor and acceptor polymers for LbL assembly include thiol-modified poly(methacrylic acid) (PMA $\left.\mathrm{PH}_{\mathrm{SH}}\right)$ and poly $(N$ vinylpyrrolidone) (PVPON), respectively. ${ }^{24-26}$ This system is attractive, as it is stable at physiological pH (disulfide cross-linking), shows pH-responsive and tunable swelling, and has permeability and degradability properties that are dependent on the choice of cross-linker. 
$\mathrm{PMA}_{\mathrm{SH}}$ capsules are obtained by the sequential deposition of PMA $\mathrm{SH}_{\mathrm{SH}}$ and PVPON at moderately acidic solutions (pH 4), followed by the oxidation of thiol groups to form disulfide linkages, and removal of the silica core particles by hydrofluoric acid (HF). ${ }^{27}$ Layering of (PVPON/PMA $\left.{ }_{\mathrm{SH}}\right)_{4} / \mathrm{PVPON}$ on $7 \mu \mathrm{m} \mathrm{SiO}_{2}$ by TFB was conducted under the optimized conditions for PAH/PSS; this yielded $\mathrm{PMA}_{\mathrm{SH}}$ capsules with no visible polymer complexation after formation of disulfide linkages, removal of PVPON and dissolution of the silica templates (Figures S3a and S4a).

Surface Morphology of TFB LbL Capsules. TFB LbL-assembled PAH/PSS capsules were characterized by fluorescence microscopy, AFM, SEM and TEM (Figure 6c-f, respectively). Under bright-field and fluorescence microscopy, the morphology of the PAH/PSS and PMA $\mathrm{PH}_{\mathrm{SH}}$ capsules assembled by TFB LbL and centrifugation-based LbL displayed typical polymer shell and hollow core characteristics. ${ }^{1,2}$ However, on closer examination by TEM and SEM, the differences in morphology between TFB LbL assembly and centrifugation-based LbL assembly were evident (Figures S3 and S4). TEM and SEM demonstrated that air-dried TFB LbL assembled capsules did not fold and form the same type of creases that are typical for capsules fabricated by centrifugation-based LbL assembly. Moreover, the film morphology exhibited non-uniform, clustered polymer complexes resulting in a "grainy" surface. The surface morphology of air-dried polymer capsules was further studied by AFM in tapping mode (Figure 7). 

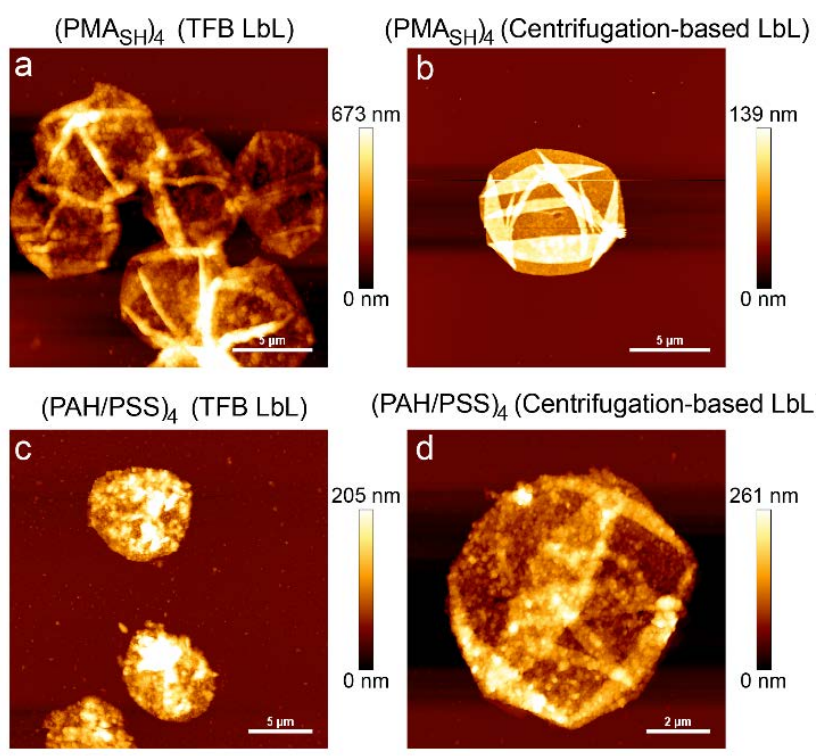

Figure 7. Atomic force microscopy (AFM) images of (PMA $\mathrm{PH}_{4}$ capsules assembled by (a) TFB and (b) centrifugation-based LbL; and (PAH/PSS) ${ }_{4}$ capsules assembled by (c) TFB and (d) centrifugation-based LbL.

The average minimum shell thickness of the film, consisting of eight $\mathrm{PMA}_{\mathrm{SH}}$ or PAH/PSS layers, corresponding to two superimposed capsule walls, was obtained from five capsules, as reported by Leporatti et al. ${ }^{28}$ Table 1 shows that while three of the systems have similar film thicknesses, TFB LbL-assembled capsules displayed a significantly rougher surface morphology. The minimum height yielded a thickness of $6.3 \pm 1.5$ and $7.2 \pm 2.4 \mathrm{~nm}$ per single PMA $\mathrm{SH}_{\mathrm{SH}}$ layer for centrifugation-based and TFB LbL assembly, respectively, and $6.6 \pm 1.3 \mathrm{~nm}$ per PAH/PSS bilayer for centrifugation-based LbL assembly. An average minimum thickness could not be determined for TFB LbL assembled (PAH/PSS) $)_{4}$ capsules, as they did not exhibit creases and folds. This value is two- to four-fold higher than that of PAH/PSS ${ }^{29}$ and $\mathrm{PMA}_{\mathrm{SH}}{ }^{30}$ capsules reported in the literature but still within the expected range for LbL-assembled capsules. 2,31,32 Root-mean-square (rms) roughness calculations over a $1.5 \mu \mathrm{m} \times 1.5 \mu \mathrm{m}$ area, free of folds, are shown in Table 1. Roughness measurements performed on TFB LbL-assembled capsules showed 
a value of $\sim 40 \mathrm{~nm}$. This value is larger than respective rms values obtained for centrifugationbased LbL capsules ( $\sim \mathrm{nm}$ for $\mathrm{PMA}_{\mathrm{SH}}$ and $\sim 20 \mathrm{~nm}$ for PAH/PSS). Previous reports have shown that increasing the polymer concentration of polyaniline $\left(\mathrm{M}_{\mathrm{W}} 20-25 \mathrm{kDa}\right)$ can increase the layer thickness and the adsorbed polymer amount with sulfonated polystyrene (SPS, $\mathrm{M}_{\mathrm{W}} 70 \mathrm{kDa}$ ). ${ }^{33}$ Additionally, the stretching of polymer $\left(\mathrm{M}_{\mathrm{W}} \sim 1\right.$ to $\left.600 \mathrm{kDa}\right)$ in the direction of the flow ${ }^{34}$ and flow-induced entanglement of the polymers ${ }^{35}$ are possible reasons for the significant increase in film roughness.

Table 1. Size, thickness and roughness of PAH/PSS and PMA ${ }_{S H}$ capsules assembled by centrifugation-based LbL and TFB LbL assembly.

\begin{tabular}{|c|c|c|c|}
\hline Capsules & $\operatorname{Size}^{\mathrm{a}}(\mu \mathrm{m})$ & Thickness $^{\mathbf{b}}(\mathrm{nm})$ & Roughness $^{\mathbf{b}}(\mathrm{nm})$ \\
\hline PAH/PSS (Centrifugation-based) & $7.1 \pm 0.3$ & $52.4 \pm 5.2$ & $22.5 \pm 3.4$ \\
\hline PAH/PSS (TFB) & $7.0 \pm 0.2$ & $-^{c}$ & $40.3 \pm 10.1$ \\
\hline $\mathrm{PMA}_{\mathrm{SH}}$ (Centrifugation-based) & $10.3 \pm 0.3$ & $50.6 \pm 5.9$ & $3.7 \pm 0.4$ \\
\hline $\mathrm{PMA}_{\mathrm{SH}}(\mathrm{TFB})$ & $8.2 \pm 0.4$ & $57.7 \pm 9.5$ & $44.5 \pm 7.7$ \\
\hline
\end{tabular}

pH and Swelling Response of TFB LbL Capsules. As capsules fabricated by TFB LbL assembly exhibited different film morphologies compared with those prepared by centrifugationbased LbL assembly, we investigated whether the material properties would differ. The $\mathrm{pH}$ responsiveness of the prepared capsules was examined by exposing the capsules to $\mathrm{NaOAc}$ buffer (pH 4) and PBS (pH 7.4) (Figure 8). The pH value was changed subsequently by washing 
the capsules with the respective buffer solutions three times before measurement. PAH/PSS capsules assembled by centrifugation-based LbL and TFB LbL were not pH-responsive after repeated $\mathrm{pH}$ cycles from $\mathrm{pH} 4$ to $\mathrm{pH}$ 7.4. As expected, this may be the result of the high degree of ionization ${ }^{36}$ of PAH $\left(\mathrm{p} K_{\mathrm{a}} \sim 8.5\right)^{37}$ and PSS $\left(\mathrm{p} K_{\mathrm{a}} \sim 1.0\right)^{38,39}$ at both acidic and neutral pHs. Combined with the high salt concentration $(0.5 \mathrm{M} \mathrm{NaCl})$ in solution, electrostatic charges between segments along the polymer are highly screened, resulting in both polyions to acquire a random coil, globule-like conformation, ${ }^{40}$ leading to a high degree of ionic cross-linking, making the interpenetrating polymer structure insensitive to swelling by $\mathrm{pH}$. However, we note that $\mathrm{pH}$ sensitive swelling has been reported by Pechekin and co-workers under highly acidic conditions (e.g., in $2.5 \mathrm{M} \mathrm{HCl})^{41}$

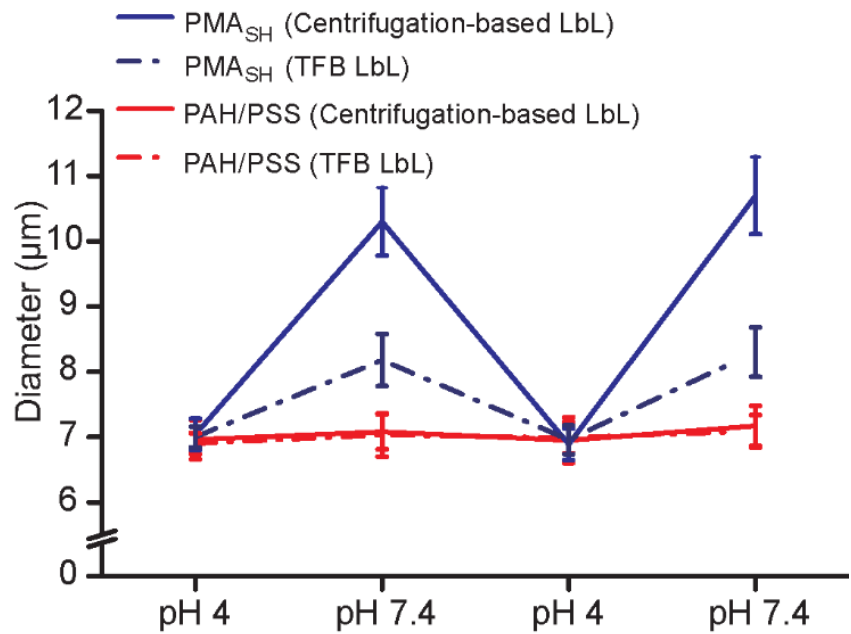

Figure 8. $\mathrm{pH}$ responsiveness of $\mathrm{PAH} / \mathrm{PSS}$ and $\mathrm{PMA}_{\mathrm{SH}}$ capsules assembled by LbL centrifugation-based or TFB. FITC-dextran-labeled and AF647-labeled capsules were cycled through $\mathrm{pH} 4$ and $\mathrm{pH} 7.4$ solutions, respectively. Capsule diameters were measured from fluorescence microscopy images. Data were averaged from at least 50 capsules. Error bars show the standard deviation of capsule diameters. Parts of the dashed red line are not visible, as they are partly behind the solid red line. 
The swelling behavior of PMA-based systems has been well documented in literature. ${ }^{26,42,43}$ Due to the carboxyl groups on the backbone of the polymer, then undergo deprotonation at physiological $\mathrm{pH}\left(\mathrm{pH}\right.$ 7.4) causing electrostatic repulsion and resulting in swelling. ${ }^{43}$ Interestingly, TFB LbL-assembled PMA $\mathrm{SH}_{\mathrm{SH}}$ capsules exhibited a lesser degree of swelling ( 20\%) compared with their centrifugation-based counterpart ( 50\%). As the degree of swelling is inversely related to the degree of cross-linking density, it may be inferred that TFB LbLassembled PMA $\mathrm{SH}_{\mathrm{SH}}$ capsules have a greater degree of cross-linking. The increase in film thickness and roughness also indicates a greater mass of polymer has been deposited through the TFB LbL process, resulting in a higher a degree of cross-linking between $\mathrm{PMA}_{\mathrm{SH}}$ chains and a corresponding reduction in film swelling. ${ }^{44}$ Consequently, this also results in a higher degree of polymer interpenetration and lower chain mobility. ${ }^{45}$

Permeability Properties of TFB LbL Capsules. Finally, we investigated the permeability of the TFB LbL capsules and compared it to those fabricated by centrifugationbased LbL assembly. All samples were incubated with a range of fluorescein isothiocyanatelabeled dextran (FITC-dextran) at pH 7.4 (PBS buffer), incubated for $30 \mathrm{~min}$, and were investigated by confocal laser scanning microscopy (CLSM) (Figure S5). (PAH/PSS) 4 capsules showed no differences in permeability, whether they are assembled by TFB or centrifugationbased LbL assembly (Figure 9). This is expected, as capsules with PAH/PSS templated on melamine formaldehyde (MF) particles are permeable to small molecules such as 6carboxyfluorescein (6-CF) ${ }^{46,47}$ and FITC-PAH. ${ }^{47}$ Moreover, even though TFB LbL-assembled PAH/PSS capsules displayed greater roughness and possibly thickness compared to their centrifugation-based LbL counterpart, it has been reported that film thickness up to (PAH/PSS)9 showed little influence over permeability of the capsules over the time scale of minutes. ${ }^{48}$ It is 
understood that the underlying microstructure of the PAH/PSS film layered at $\mathrm{pH} \sim 7$ and high salt concentration results in sufficient porosity to allow unhindered diffusion of molecules through the capsule shell. Nevertheless, the PAH/PSS system has demonstrated tunable permeability through changes in $\mathrm{pH}^{49}$ salt concentration ${ }^{50}$ and solvent. ${ }^{51}$ However, $\mathrm{PMA}_{\mathrm{SH}}$ capsules assembled by TFB LbL have reduced permeability compared to centrifugation-based LbL capsules with the molecular weight cutoff point reduced from 59-77 kDa to $20 \mathrm{kDa}$ FITCdextran (Figure S5), which is a critical size range for retention of therapeutic cargo, such as insulin hexamers and lipase. ${ }^{52,53}$ As already observed in the AFM data and swelling studies, TFB PMA $_{S H}$ capsules have increased polymer deposition and reduced swelling, which indicates a decrease in mesh size of the polymer film. Miller and co-workers demonstrated a similar relationship where the rejection of water soluble sugar units generally increases as the swelling of the polyelectrolyte film decreases. ${ }^{45}$ According to our data, polymer films stabilized by hydrogen bonding and layered under flow conditions have higher film thickness and roughness values, exhibiting reduced permeability toward permeation of dextrans in comparison to PAH/PSS films. Therefore, TFB LbL assembly addresses key challenges associated with fluidized bed LbL assembly, while retaining advantages such as ease-of-use, high amenability to automation, and potential for scale-up. 


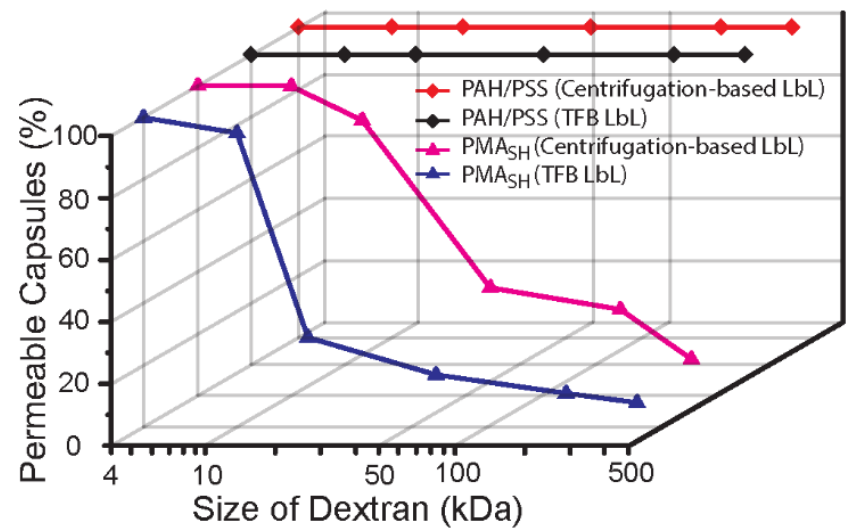

Figure 9. Permeability of PAH/PSS and PMA $\mathrm{PH}_{\mathrm{SH}}$ capsules using 4, 10, 20, 59-77, 250 and 500 kDa FITC-dextran.

\section{CONCLUSIONS}

This study demonstrates that LbL capsules can be prepared by TFB LbL assembly through the use of directional flow for depositing different polymer coatings onto fluidized template particles for the formation of microcapsules. It was found that theoretical $\dot{\mathrm{V}}_{\mathrm{MF}}$ and $\dot{\mathrm{V}}_{\mathrm{T}}$ values calculated based on the minimum and maximum diameter of the tapered column design corresponded well to the experimentally determined values. TFB LbL assembly was capable of fluidizing differentsized particles over a wide range of flow rates and yields stable capsules for layering charged and hydrogen-bonding polymers. The shell thickness of the capsules prepared via TFB LbL assembly is similar to those prepared by CFB LbL assembly, which is two- to four-fold greater than centrifugation-based LbL. Moreover, TFB LbL-assembled capsules exhibited different surface morphology and properties compared to their centrifugation-based counterparts, possessing greater film thickness and roughness. Importantly, $\mathrm{PMA}_{\mathrm{SH}}$ capsules also exhibit less swelling at physiological $\mathrm{pH}$ and reduced permeability to fluorescently labeled dextrans, improving the capacity to encapsulate small ( $20 \mathrm{kDa}$ ) molecules, which may be useful for applications ranging from biological sensing to controlled drug delivery of therapeutic cargo such as small proteins. 
We also note that particulate templates of different composition, such as gold, and different shapes should be equally applicable to TFB.

\section{ASSOCIATED CONTENT}

Supporting Information. Additional experimental details, TEM and SEM images, and CLSM images of capsule permeability. This material is available free of charge via the Internet at http://pubs.acs.org.

\section{AUTHOR INFORMATION}

\section{Corresponding Author}

*Email: fcaruso@unimelb.edu.au

\section{Notes}

The authors declare no competing financial interest.

$\ddagger$ Present address: CSIRO Manufacturing Flagship, CSIRO Private Bag 10, Clayton South, Victoria 3169, Australia

\section{Author Contributions}

The manuscript was written through contributions of all authors. All authors have given approval to the final version of the manuscript.

\section{ACKNOWLEDGMENT}

This research was conducted and funded by the Australian Research Council (ARC) Centre of Excellence in Convergent Bio-Nano Science and Technology (Project CE140100036) and the ARC under the Australian Laureate Fellowship (FL120100030) scheme, and by the Australian Government through an Australian Postgraduate Award (M.B.). This work was performed in part at the Materials Characterisation and Fabrication Platform (MCFP) at the University of 
Melbourne and the Victorian Node of the Australian National Fabrication Facility (ANFF). The authors acknowledge Prof. Ray Dagastine, the University of Melbourne, for helpful discussions.

\section{REFERENCES}

1. Decher, G. Fuzzy Nanoassemblies: Toward Layered Polymeric Multicomposites. Science 1997, 277, 1232-1237.

2. Richardson, J. J.; Björnmalm, M.; Caruso, F. Technology-Driven Layer-by-Layer Assembly of Nanofilms. Science 2015, 348, aaa2491.

3. Elbakry, A.; Zaky, A.; Liebl, R.; Rachel, R.; Goepferich, A.; Breunig, M. Layer-by-Layer Assembled Gold Nanoparticles for siRNA Delivery. Nano Lett. 2009, 9, 2059-2064.

4. Caruso, F.; Caruso, R. A.; Möhwald, H. Nanoengineering of Inorganic and Hybrid Hollow Spheres by Colloidal Templating. Science 1998, 282, 1111-1114.

5. Calvo, P.; Remuñán-López, C.; Vila-Jato, J. L.; Alonso, M. J. Development of Positively Charged Colloidal Drug Carriers: Chitosan-Coated Polyester Nanocapsules and SubmicronEmulsions. Colloid Polym. Sci. 1997, 275, 46-53.

6. Tong, W.; Song, X.; Gao, C. Layer-by-Layer Assembly of Microcapsules and Their Biomedical Applications. Chem. Soc. Rev. 2012, 41, 6103-6124.

7. Lvov, Y.; Ariga, K.; Ichinose, I.; Kunitake, T. Assembly of Multicomponent Protein Films by Means of Electrostatic Layer-by-Layer Adsorption. J. Am. Chem. Soc. 1995, 117, 61176123.

8. Decher, G.; Lehr, B.; Lowack, K.; Lvov, Y.; Schmitt, J. New Nanocomposite Films for Biosensors: Layer-by-Layer Adsorbed Films of Polyelectrolytes, Proteins or DNA. Biosens. Bioelectron. 1994, 9, 677-684.

9. $\quad$ Iler, R. K. Multilayers of Colloidal Particles. J. Colloid Interface Sci. 1966, 21, 569-594. 
10. Voigt, A.; Lichtenfeld, H.; Sukhorukov, G. B.; Zastrow, H.; Donath, E.; Bäumler, H.; Möhwald, H. Membrane Filtration for Microencapsulation and Microcapsules Fabrication by Layer-by-Layer Polyelectrolyte Adsorption. Ind. Eng. Chem. Res. 1999, 38, 4037-4043.

11. Schlenoff, J. B.; Dubas, S. T.; Farhat, T. Sprayed Polyelectrolyte Multilayers. Langmuir 2000, 16, 9968-9969.

12. Thomas, I. M. Single-Layer $\mathrm{TiO}_{2}$ and Multilayer $\mathrm{TiO}_{2}-\mathrm{SiO}_{2}$ Optical Coatings Prepared from Colloidal Suspensions. Appl. Opt. 1987, 26, 4688-4691.

13. Qi, A.; Chan, P.; Ho, J.; Rajapaksa, A.; Friend, J.; Yeo, L. Template-Free Synthesis and Encapsulation Technique for Layer-by-Layer Polymer Nanocarrier Fabrication. ACS Nano 2011, 5, 9583-9591.

14. Richardson, J. J.; Liang, K.; Kempe, K.; Ejima, H.; Cui, J.; Caruso, F. Immersive Polymer Assembly on Immobilized Particles for Automated Capsule Preparation. Adv. Mater. 2013, 25, 6874-6878.

15. Kantak, C.; Beyer, S.; Yobas, L.; Bansal, T.; Trau, D. A 'Microfluidic Pinball' for OnChip Generation of Layer-by-Layer Polyelectrolyte Microcapsules. Lab Chip 2011, 11, 10301035.

16. Richardson, J. J.; Ejima, H.; Lörcher, S. L.; Liang, K.; Senn, P.; Cui, J.; Caruso, F. Preparation of Nano- and Microcapsules by Electrophoretic Polymer Assembly. Angew. Chem. Int. Ed. 2013, 52, 6455-6458.

17. Richardson, J. J.; Teng, D.; Björnmalm, M.; Gunawan, S. T.; Guo, J.; Cui, J.; Franks, G. V.; Caruso, F. Fluidized Bed Layer-by-Layer Microcapsule Formation. Langmuir 2014, 30, 10028-10034. 
18. San Jose, M. J.; Olazar, M.; Penas, F. J.; Bilbao, J. Segregation in Conical Spouted Beds with Binary and Ternary Mixtures of Equidensity Spherical Particles. Ind. Eng. Chem. Res. 1994, 33, 1838-1844.

19. Permchart, W.; Kouprianov, V. I. Emission Performance and Combustion Efficiency of a Conical Fluidized-Bed Combustor Firing Various Biomass Fuels. Bioresour. Technol. 2004, 92, 83-91.

20. Rao, D. G.; Senthilkumar, R.; Byrne, J. A.; Feroz, S. Wastewater Treatment: Advanced Processes and Technologies. Taylor \& Francis: Oxford, UK, 2012.

21. Scott, C. D.; Hancher, C. W. Use of a Tapered Fluidized Bed as a Continuous Bioreactor. Biotechnol. Bioeng. 1976, 18, 1393-1403.

22. Shi, Y. F.; Yu, Y.; Fan, L. Incipient Fluidization Condition for a Tapered Fluidized Bed. Ind. Eng. Chem. Fundam. 1984, 23, 484-489.

23. Kempe, K.; Noi, K. F.; Ng, S. L.; Müllner, M.; Caruso, F. Multilayered Polymer Capsules with Switchable Permeability. Polymer 2014, 55, 6451-6459.

24. Zelikin, A. N.; Li, Q.; Caruso, F. Degradable Polyelectrolyte Capsules Filled with Oligonucleotide Sequences. Angew. Chem. Int. Ed. 2006, 45, 7743-7745.

25. Zelikin, A. N.; Li, Q.; Caruso, F. Disulfide-Stabilized Poly(Methacrylic Acid) Capsules: Formation, Cross-Linking, and Degradation Behavior. Chem. Mater. 2008, 20, 2655-2661.

26. Kozlovskaya, V.; Kharlampieva, E.; Mansfield, M. L.; Sukhishvili, S. A. Poly(methacrylic acid) Hydrogel Films and Capsules: Response to $\mathrm{pH}$ and Ionic Strength, and Encapsulation of Macromolecules. Chem. Mater. 2006, 18, 328-336.

27. Zelikin, A. N.; Quinn, J. F.; Caruso, F. Disulfide Cross-Linked Polymer Capsules: En Route to Biodeconstructible Systems. Biomacromolecules 2006, 7, 27-30. 
28. Leporatti, S.; Voigt, A.; Mitlöhner, R.; Sukhorukov, G.; Donath, E.; Möhwald, H. Scanning Force Microscopy Investigation of Polyelectrolyte Nano- and Microcapsule Wall Texture. Langmuir 2000, 16, 4059-4063.

29. Sukhorukov, G. B.; Donath, E.; Lichtenfeld, H.; Knippel, E.; Knippel, M.; Budde, A.; Möhwald, H. Layer-by-Layer Self Assembly of Polyelectrolytes on Colloidal Particles. Colloids Surf., A 1998, 137, 253-266.

30. Becker, A. L.; Zelikin, A. N.; Johnston, A. P. R.; Caruso, F. Tuning the Formation and Degradation of Layer-by-Layer Assembled Polymer Hydrogel Microcapsules. Langmuir 2009, 25, 14079-14085.

31. De Geest, B. G.; Déjugnat, C.; Prevot, M.; Sukhorukov, G. B.; Demeester, J.; De Smedt, S. C. Self-Rupturing and Hollow Microcapsules Prepared from Bio-Polyelectrolyte-Coated Microgels. Adv. Funct. Mater. 2007, 17, 531-537.

32. Sukhishvili, S. A.; Granick, S. Layered, Erasable Polymer Multilayers Formed by Hydrogen-Bonded Sequential Self-Assembly. Macromolecules 2002, 35, 301-310.

33. Cheung, J. H.; Stockton, W. B.; Rubner, M. F. Molecular-Level Processing of Conjugated Polymers. 3. Layer-by-Layer Manipulation of Polyaniline via Electrostatic Interactions. Macromolecules 1997, 30, 2712-2716.

34. Evans, A. R.; Shaqfeh, E. S. G.; Frattini, P. L. Observations of Polymer Conformation during Flow through a Fixed Fibre Bed. J. Fluid Mech. 1994, 281, 319-356.

35. Hikmet, R. A. M.; Narh, K. A.; Barham, P. J.; Keller, A. Adsorption-Entanglement Layers in Flowing High-Molecular Weight Polymer Solutions. In Frontiers in Polymer Science, Wilke, W., Ed. Steinkopff: 1985; Chapter 6, pp 32-43. 
36. Smith, R. N.; McCormick, M.; Barrett, C. J.; Reven, L.; Spiess, H. W. NMR Studies of PAH/PSS Polyelectrolyte Multilayers Adsorbed onto Silica. Macromolecules 2004, 37, 48304838.

37. Choi, J.; Rubner, M. F. Influence of the Degree of Ionization on Weak Polyelectrolyte Multilayer Assembly. Macromolecules 2005, 38, 116-124.

38. Li, L.; Ferng, L.; Wei, Y.; Yang, C.; Ji, H.-F. Effects of Acidity on the Size of Polyaniline-Poly(Sodium 4-Styrenesulfonate) Composite Particles and the Stability of Corresponding Colloids in Water. J. Colloid Interface Sci. 2012, 381, 11-16.

39. Hua, F.; Shi, J.; Lvov, Y.; Cui, T. Fabrication and Characterization of Metal-OxideSemiconductor Capacitor Based on Layer-by-Layer Self-Assembled Thin Films. Nanotechnology 2003, 14, 453.

40. Ladam, G.; Schaad, P.; Voegel, J. C.; Schaaf, P.; Decher, G.; Cuisinier, F. In Situ Determination of the Structural Properties of Initially Deposited Polyelectrolyte Multilayers. Langmuir 2000, 16, 1249-1255.

41. Pechenkin, M. A.; Möhwald, H.; Volodkin, D. V. pH- and Salt-Mediated Response of Layer-by-Layer Assembled PSS/PAH Microcapsules: Fusion and Polymer Exchange. Soft Matter 2012, 8, 8659-8665.

42. Chong, S.-F.; Lee, J. H.; Zelikin, A. N.; Caruso, F. Tuning the Permeability of Polymer Hydrogel Capsules: An Investigation of Cross-Linking Density, Membrane Thickness, and Cross-Linkers. Langmuir 2011, 27, 1724-1730.

43. Zhang, J.; Peppas, N. A. Synthesis and Characterization of $\mathrm{pH}-$ and TemperatureSensitive Poly(methacrylic acid)/Poly( $N$-isopropylacrylamide) Interpenetrating Polymeric Networks. Macromolecules 2000, 33, 102-107. 
44. Zhuk, A.; Mirza, R.; Sukhishvili, S. Multiresponsive Clay-Containing Layer-by-Layer Films. ACS Nano 2011, 5, 8790-8799.

45. Miller, M. D.; Bruening, M. L. Correlation of the Swelling and Permeability of Polyelectrolyte Multilayer Films. Chem. Mater. 2005, 17, 5375-5381.

46. Caruso, F.; Donath, E.; Möhwald, H. Influence of Polyelectrolyte Multilayer Coatings on Förster Resonance Energy Transfer between 6-Carboxyfluorescein and Rhodamine B-Labeled Particles in Aqueous Solution. J. Phys. Chem. B 1998, 102, 2011-2016.

47. Sukhorukov, G. B.; Donath, E.; Moya, S.; Susha, A. S.; Voigt, A.; Hartmann, J.; Möhwald, H. Microencapsulation by Means of Step-Wise Adsorption of Polyelectrolytes. J. Microencapsulation 2000, 17, 177-185.

48. Antipov, A. A.; Sukhorukov, G. B. Polyelectrolyte Multilayer Capsules as Vehicles with Tunable Permeability. Adv. Colloid Interface Sci. 2004, 111, 49-61.

49. Antipov, A. A.; Sukhorukov, G. B.; Leporatti, S.; Radtchenko, I. L.; Donath, E.; Möhwald, H. Polyelectrolyte Multilayer Capsule Permeability Control. Colloids Surf., A 2002, 198-200, 535-541.

50. Ibarz, G.; Dähne, L.; Donath, E.; Möhwald, H. Smart Micro- and Nanocontainers for Storage, Transport, and Release. Adv. Mater. 2001, 13, 1324-1327.

51. Lvov, Y.; Antipov, A. A.; Mamedov, A.; Möhwald, H.; Sukhorukov, G. B. Urease Encapsulation in Nanoorganized Microshells. Nano Lett. 2001, 1, 125-128.

52. De Caro, A.; Figarella, C.; Amic, J.; Michel, R.; Guy, O. Human Pancreatic Lipase: A Glycoprotein. Biochim. Biophys. Acta, Protein Struct. 1977, 490, 411-419.

53. Fabris, D.; Fenselau, C. Characterization of Allosteric Insulin Hexamers by Electrospray Ionization Mass Spectrometry. Anal. Chem. 1999, 71, 384-387. 


\section{TABLE OF CONTENTS GRAPHIC}

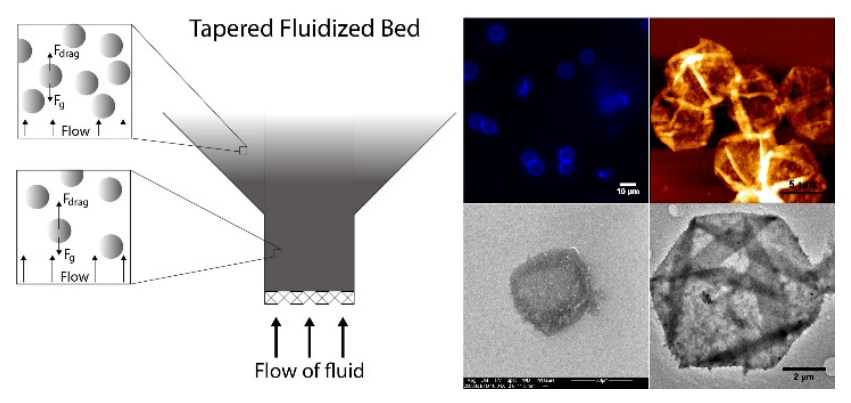

